\title{
Hyperhomocysteinemia Associated with Low Muscle Mass, Muscle Function in Elderly Hemodialysis Patients: An Analysis of Multiple Dialysis Centers
}

\author{
Chi-Sin Wang, ${ }^{1}$ Te-Chih Wong, ${ }^{2}$ Tuyen Van Duong $\mathbb{D}^{1},{ }^{1}$ Chien-Tien Su $\mathbb{D}^{1},{ }^{3,4}$ \\ Hsi-Hsien Chen $\left(\mathbb{D},{ }^{5,6}\right.$ Tso-Hsiao Chen, ${ }^{5,7}$ Yung-Ho Hsu, ${ }^{5,8}$ Sheng-Jeng Peng, ${ }^{9}$ Ko-Lin Kuo, ${ }^{10}$ \\ Hsiang-Chung Liu, ${ }^{11}$ En-Tzu Lin, ${ }^{12}$ Yi-Wei Feng, ${ }^{1}$ and Shwu-Huey Yang ${ }^{10}{ }^{1,13,14}$ \\ ${ }^{1}$ School of Nutrition and Health Sciences, Taipei Medical University, Taipei, Taiwan \\ ${ }^{2}$ Department of Nutrition and Health Sciences, Chinese Culture University, Taipei, Taiwan \\ ${ }^{3}$ School of Public Health, Taipei Medical University, Taipei, Taiwan \\ ${ }^{4}$ Department of Family Medicine, Taipei Medical University Hospital, Taipei, Taiwan \\ ${ }^{5}$ Division of Nephrology, Department of Internal Medicine, School of Medicine, College of Medicine, Taipei Medical University, \\ Taipei, Taiwan \\ ${ }^{6}$ Division of Nephrology, Department of Internal Medicine, Taipei Medical University Hospital, Taipei, Taiwan \\ ${ }^{7}$ Department of Nephrology, Taipei Medical University-Wan Fang Hospital, Taipei, Taiwan \\ ${ }^{8}$ Division of Nephrology, Department of Internal Medicine, Taipei Medical University-Shuang Ho Hospital, Taipei, Taiwan \\ ${ }^{9}$ Division of Nephrology, Cathay General Hospital, Taipei, Taiwan \\ ${ }^{10}$ Division of Nephrology, Taipei Tzu-Chi Hospital, Taipei, Taiwan \\ ${ }^{11}$ Department of Nephrology, Wei Gong Memorial Hospital, Miaoli, Taiwan \\ ${ }^{12}$ Department of Nephrology, Lotung Poh-Ai Hospital, Yilan, Taiwan \\ ${ }^{13}$ Research Center of Geriatric Nutrition, Taipei Medical University, Taipei, Taiwan \\ ${ }^{14}$ Nutrition Research Center, Taipei Medical University Hospital, Taipei, Taiwan
}

Correspondence should be addressed to Shwu-Huey Yang; sherry@tmu.edu.tw

Received 22 March 2019; Accepted 23 May 2019; Published 9 June 2019

Academic Editor: Germán Vicente-Rodriguez

Copyright (C) 2019 Chi-Sin Wang et al. This is an open access article distributed under the Creative Commons Attribution License, which permits unrestricted use, distribution, and reproduction in any medium, provided the original work is properly cited.

Background. The hyperhomocysteinemia was with high prevalence and has been considered as a risk factor for cardiovascular disease in hemodialysis patients. These patients also experienced a high risk of muscle wasting caused by the comorbidity, malnutrition, and low physical activity. We investigated the associations of homocysteinemia with muscle mass, muscle function in elderly hemodialysis patients. Methods. A clinical cross-sectional study was conducted on 138 hemodialysis patients aged 65 years and above in seven hospital-based hemodialysis centers in Taiwan. The data on anthropometry, laboratory, and 3day dietary intake was examined. The skeletal muscle mass (SMM) was measured by the bioelectrical impedance analysis; the $\mathrm{SMM}$ was adjusted by height or weight as $\mathrm{SMM}_{\mathrm{Ht} 2}\left(\mathrm{~kg} / \mathrm{m}^{2}\right)$ and $\mathrm{SMM}_{\mathrm{Wt}}(\%)$. Muscle function was defined as handgrip strength (HGS) (kg) measured by handgrip dynamometer. Statistical analyses were conducted using simple regression and multivariable stepwise regression analysis. Results. In the total sample, $74.6 \%$ of hemodialysis patients were hyperhomocysteinemia ( $\geq 15$ $\mu \mathrm{mol} / \mathrm{L}$ ). The means of $\mathrm{SMM}_{\mathrm{Ht} 2}, \mathrm{SMM}_{\mathrm{Wt}}$, arm lean mass, hand grip strength, and muscle quality were 8.7 $\pm 1.2,37.7 \pm 5.6,1.7$ $\pm 0.5,21.1 \pm 7.4$, and $10.0 \pm 3.0$, respectively. The multivariable stepwise regression analysis showed that homocysteinemia level was significantly inversely associated with $\mathrm{SMM}_{\mathrm{Wt}}(\mathrm{B}$-coeff. $=-0.03, p=0.02)$ in hemodialysis patients above 65 years old, but not with muscle function. Conclusions. Hyperhomocysteinemia is common and associated with decreased muscle mass in the elderly hemodialysis patients. Future studies are suggested to explore the impact of the homocysteine-lowering therapy on muscle decline. 


\section{Introduction}

The United States Renal Data System reported that the prevalence of treated end-stage renal disease (ESRD) was highest for individuals aged 65-74 years in Taiwan [1]. The elderly hemodialysis patients experienced the high risk for muscle wasting, which was related to higher morbidity and mortality [2]. Muscle wasting was the most common condition in elderly people, especially those undergoing hemodialysis with a high prevalence of decreased muscle mass and muscle function, varying from $12.7 \%$ to $45.1 \%$ in hemodialysis patients [3], the declines in muscle mass and function caused by age [3], comorbidity like diabetes mellitus and infections [4], malnutrition [5], and physical inactivity [6]. In addition, physicians prescribed exercise restrictions in those patients with complications during the treatment [7]. Furthermore, declines of muscle mass and function were exaggerated due to the long bedridden time for the dialysis, low physical function [8], low exercise capacity [9], and increased muscle atrophy [6].

The hyperhomocysteinemia was presented in $80 \%$ to $90 \%$ of hemodialysis cases [10]. Homocysteine (Hcy) has been considered an important cardiovascular risk factor [11]. Moreover, studies have established an association between high levels of homocysteine and a decline in physical function in elderly populations [12]. The lower muscle mass was observed in higher plasma homocysteine group in $\geq 65$ years adults [13]. The effects of hyperhomocysteinemia in vascular and myocyte function leading to impaired muscle function were summarized as follows: (i) oxidative defense reduced and production of reactive oxygen species enhanced, (ii) inhibition of nitric oxide (NO) signaling, (iii) inflammation and its associated changes, and (vi) endoplasmic reticulum (ER) stress enhanced [14]. Previous empirical studies have shown elevated Hcy associated with the decline of muscle strength and physical function in the older adults $[15,16]$.

Since hemodialysis patients are with a high prevalence of hyperhomocysteinemia and in a high-risk group of muscle wasting, however, there are a limited number of studies that discussed this issue in hemodialysis patients, especially in the elderly. Therefore, we aim to investigate the association between homocysteine level and muscle mass, muscle function in elderly hemodialysis patients.

\section{Materials and Methods}

2.1. Study Design and Patients. A clinical cross-sectional design was conducted from September 2013 to April 2017 in seven hospital-based hemodialysis centers in Taiwan. The study was approved by the Taipei Medical University Joint Institutional Review Board (no. TMU-JIRB 201302024) for conducting in Taipei Medical University Hospital, Taipei Medical University-Wan Fang Hospital, Taipei Medical University-Shuang Ho Hospital, Wei Gong Memorial Hospital, and Lotung Poh-Ai Hospital, the institutional Ethics Committee from Cathay General Hospital (no. CGHOP104001), and Taipei Tzu-Chi Hospital (no. 04-M11-090).
All patients signed written informed consent forms before their participation.

The total sample of 138 patients aged 65 years and above and undergoing hemodialysis was recruited for the study. Patients who received stable hemodialysis treatment in the previous 3 months with equilibrated $\mathrm{Kt} / \mathrm{V}$ of 1.2 and higher were included. Patients with obvious edema, hyperthyroidism, hypothyroidism, amputation, malign tumor, pregnancy, or hospitalization for renal disease reason were excluded.

\subsection{Data Collection}

2.2.1. Demographics Data. We conducted chart reviews to collect the data related to age, gender, dialysis vintage, diabetes mellitus (DM), hypertension, cardiovascular disease (CVD), and the anthropometry data including dry weight, height, and interdialytic weight gain.

2.2.2. Physical Activity. Patients' physical activity was assessed using the short version of the International Physical Activity Questionnaire (IPAQ) [17]. Interviewers recorded the average number of days per week and the average time per day that patient spent on exercising (vigorous, moderate, or walking exercise) in the past 7 days. The value of metabolic equivalent (MET in $\mathrm{kcal} / \mathrm{day}$ ) value was used to examine the levels of physical activity [17].

2.2.3. Dietary Intake Data. All patients wrote down a threeday dietary record, including one dialysis day, one nondialysis day, and one day during the weekend. The data collection of dietary intake was also mentioned in our previous publication [18]. In brief, qualified dietitians taught patients how to fill in the record. To assure the record, the well-trained dietitians contacted with all patients and conducted the interviews by face-to-face, or by telephone. Next, the dietitians used the $24 \mathrm{~h}$ recall to confirm the data provided by patients using the common utensils in the household as the means. The nutrients were then analyzed using nutrients analysis software (e-Kitchen, Taichung, Taiwan) based on Taiwanese nutrition compositions as the nutrient database.

2.2.4. Biochemical Values. We collected the 8-hour fasting and predialysis blood samples and sent to the Laboratory Department in Taipei Medical University Hospital for analyzing the biochemical parameters. The following parameters were collected by reviewing patient medical charts: total cholesterol, creatinine, and fasting blood glucose.

\subsection{Measurements}

2.3.1. Homocysteine Measurements. Homocysteine was measured used in the enzymatic method. 8-hour fasting and predialysis blood samples were withdrawn and collected in EDTA blood collection tubes keep tube cold at $4^{\circ} \mathrm{C}$ and centrifuged within 1-2 hour, then plasma was assessed using the Roche Cobas c702 automatic analyzer (Rui An international 
Co. Ltd., Taipei, Taiwan) at the of the Laboratory Department in Taipei Medical University Hospital.

2.3.2. Other Biochemical Measurements. The 8-hour fasting and predialysis blood samples were analyzed using standardized protocols for serum albumin, insulin, serum folate, serum vitamin $B_{12}$, high-sensitivity C-reactive protein (hsCRP), thyroid-stimulating hormone, and plasma homocysteine. The cutoff values for folate and $B_{12}$ were $3 \mathrm{ng} / \mathrm{mL}$ and $250 \mathrm{pg} / \mathrm{mL}$, respectively. Additionally, insulin resistance was measured by the homeostasis model assessmentestimated insulin resistance (HOMA-IR) calculated as (glucose $[\mathrm{mg} / \mathrm{dL}] \times$ insulin $[\mu \mathrm{U} / \mathrm{mL})] / 405$ [19]. In the clinical assessment of nutritional status, we calculated the geriatric nutritional risk index (GNRI) as described previously, when GNRI $<91.2$ exhibited a poorer nutritional status. Hypoalbuminemia and inflammation were defined as serum albumin less than $3.5 \mathrm{~g} / \mathrm{dL}$ and hs-CRP higher than $0.5 \mathrm{mg} / \mathrm{dL}$, respectively. Normalized protein nitrogen appearance (nPNA) $\geq 1.2$ $\mathrm{g} / \mathrm{kg}$ was appropriate protein intake in hemodialysis patients as suggested by the National Kidney Foundation [20].

2.3.3. Anthropometry Measurements. Skeletal muscle mass (SMM) and body fat are measured by using the bioelectrical impedance analysis (InBody S10, Biospace, Seoul, Korea) after the hemodialysis session (sitting position). The eight surface electrodes are placed on the thumbs, middle fingers, and either side of the ankles of the patients using multiple operating frequencies of $1,5,50,250,500$, and 1,000 kHz. Moreover, SMM was normalized for weight as $\operatorname{SMM}_{\mathrm{Wt}}(\%)$ and height as $\mathrm{SMM}_{\mathrm{Ht} 2}\left(\mathrm{~kg} / \mathrm{m}^{2}\right)$, which are indicators of muscle mass. Muscle quality (MQ) is an important determinant of muscle function, defined as muscle strength or power per unit of muscle mass and calculated as the ratio of hand grip strength $(\mathrm{kg})$ to arm lean mass $(\mathrm{kg})$ [21]. The handgrip strength (HGS) was measured before a hemodialysis session using grip strength dynamometer (Jamar, Sammons Preston, Bolingbrook, IL) with a precision of $0.5 \mathrm{~kg}$. During the measurement, patients were asked to stand straight with arm and hands being neutrally hung beside the body and then use maximum effort to squeeze the dynamometer with nonfistula hand for at least 3 seconds, and each time was provided at least 10 seconds for recovery. Patient performed the handgrip for 3 times and the pick performance was noted as the final result.

2.4. Statistical Analyses. The sample of 138 patients with data of muscle mass and a sample of 87 patients with the data of muscle function were analyzed. The descriptive analyses were used to illustrate the mean \pm standard deviation, percentage of social demographics, biochemical parameters, dietary intake, and patients' characteristics. Simple regression was used to identify the predictors of muscle mass and muscle function which were known as prognostic factors, statistical significance when $\mathrm{p}<0.05$. Multivariate linear regression models were performed to investigate the associations of Hcy and the muscle mass and muscle function. The variables showed the associations with muscle mass and function at $\mathrm{p}$ $<0.2$ in the simple regression analyses which were kept in the multivariate models. Variables such as age, gender, and energy intake were also included in the analysis. The unstandardized regression coefficient (B), 95\% confidence interval, and adjusted $\mathrm{R}$ square values were presented appropriately. Statistical analyses were performed using SAS software (ver. 9.4; SAS Institute Inc., Cary, NC, USA).

\section{Results}

3.1. Characteristics of Hemodialysis Patients. Demographics, anthropometrics, biochemical parameters, and clinical information are shown in Table 1 . In 138 hemodialysis patients, $63.0 \%$ are men; $47.1 \%, 51.5 \%$, and $43.5 \%$ of the hemodialysis patients had DM, hypertension, and a history of CVD, respectively. The means of age and hemodialysis vintage were $73.1 \pm 6.4$ years and $4.5 \pm 3.4$ years, respectively. The plasma Hcy level was $19.7 \pm 6.0 \mu \mathrm{mol} / \mathrm{L} ; 74.6 \%$ of hemodialysis patients present in hyperhomocysteinemia $(\geq 15 \mu \mathrm{mol} / \mathrm{L}$ ). The serum folate and vitamin $B_{12}$ were $18.1 \pm 4.5 \mathrm{ng} / \mathrm{mL}$ and $1416.8 \pm 582.6 \mathrm{pg} / \mathrm{mL}$, respectively. Hemodialysis patients were in a healthier and had good nutritional status, according to the GNRI $98.6 \pm 7.1$, serum albumin $3.9 \pm 0.4 \mathrm{~g} / \mathrm{dL}$, and nPNA $1.3 \pm 0.4 \mathrm{~g} /$ day, respectively (Table 1 ).

3.2. Associated Factors of Muscle Mass and Muscle Function. The SMM, $\mathrm{SMM}_{\mathrm{Ht}}$, and $\mathrm{SMM}_{\mathrm{Wt}}$ were positively correlated with creatinine and height and were negatively correlated with age, gender, and body fat. HGS was positively associated with creatinine and height and was negatively correlated with gender. Muscle quality (MQ) was negatively correlated with body weight and BMI (Table 2).

The results stepwise regressions show that SMM was significant associations with energy intake (regression coefficient, $\mathrm{B}=<0.01)$, $\mathrm{BMI}(\mathrm{B}=1.03)$, and body fat $(\mathrm{B}=-0.37)$ (adjusted $\mathrm{R}^{2}=0.82$ ), $\mathrm{SMM}_{\mathrm{H} 2}$ was significant associations with creatinine $(B=0.23)$ and body fat $(B=-0.04)$ (adjusted $\left.\mathrm{R}^{2}=0.50\right), \mathrm{SMM}_{\mathrm{Wt}}$ was significant associations with Hcy $(B=-0.03)$, creatinine $(B=0.13)$, and body fat $(B=-0.57)$ (adjusted $\mathrm{R}^{2}=0.97$ ) (Table 3). In muscle function, HGS was significant associations with creatinine $(B=0.93)$ (adjusted $\left.\mathrm{R}^{2}=0.35\right)$; MQ was significant associations with creatinine $(\mathrm{B}=0.45)$ and $\mathrm{BMI}(\mathrm{B}=-0.58)$ (adjusted $\left.\mathrm{R}^{2}=0.18\right)$. Plasma Hcy level was the independent risk determinants of $S M_{\mathrm{Wt}}$ in hemodialysis patients $\geq 65$ years old (adjusted $\mathrm{R}^{2}=0.97$, $\mathrm{p}$ $=0.02$ ).

After controlling the analysis subgroup of hyperhomocysteinemia hemodialysis patients, Hcy was significantly associated with $\mathrm{SMM}_{\mathrm{Wt}}(\mathrm{B}=-0.03, \mathrm{p}=0.027)$ (adjusted $\mathrm{R}^{2}$ $=0.99)$, HGS $(B=-0.41, p=0.058)$ (adjusted $\left.R^{2}=0.35\right)$, and MQ ( $\mathrm{B}=-0.18, \mathrm{p}=0.071)$ (adjusted $\left.\mathrm{R}^{2}=0.19\right)$ in hyperhomocysteinemia hemodialysis patients (Table 4).

\section{Discussion}

The current study found that Hcy was significantly inversely associated with muscle mass in hemodialysis patients aged 65 
TABle 1: Demographic, anthropometric, dietary, biochemical values, indicators of muscle mass, and muscle function in hemodialysis patients.

\begin{tabular}{|c|c|}
\hline Variables & All $(n=138)$ \\
\hline \multicolumn{2}{|l|}{ Demographics } \\
\hline Age (years) & $73.1 \pm 6.4$ \\
\hline Male & $87(63.0 \%)$ \\
\hline Dialysis vintage (year) & $4.5 \pm 3.4$ \\
\hline Interdialytic weight gain (\%) & $3.3 \pm 1.2$ \\
\hline $\mathrm{eKt} / \mathrm{V}$ & $1.6 \pm 0.3$ \\
\hline Diabetes mellitus & $65(47.1 \%)$ \\
\hline Hypertension & $71(51.5 \%)$ \\
\hline History of CVD & $60(43.5 \%)$ \\
\hline \multicolumn{2}{|l|}{ Biochemical values } \\
\hline Albumin (g/dL) & $3.9 \pm 0.4$ \\
\hline Total cholesterol (mg/dL) & $161.7 \pm 34.8$ \\
\hline Creatinine (mg/dL) & $10.5 \pm 1.8$ \\
\hline $\mathrm{FPG}(\mathrm{mg} / \mathrm{dL})$ & $137.7 \pm 63.2$ \\
\hline Insulin $(\mu \mathrm{U} / \mathrm{mL})$ & $23.6 \pm 22.4$ \\
\hline HOMA-IR & $9.1 \pm 12.3$ \\
\hline Homocysteine $(\mu \mathrm{mol} / \mathrm{L})$ & $19.7 \pm 6.0$ \\
\hline Hyperhomocysteinemia & $103(74.6 \%)$ \\
\hline Folate (ng/mL) & $18.1 \pm 4.5$ \\
\hline Vitamin $B_{12}(\mathrm{pg} / \mathrm{mL})$ & $1416.8 \pm 582.6$ \\
\hline $\mathrm{TSH}(\mu \mathrm{U} / \mathrm{mL})$ & $2.5 \pm 2.1$ \\
\hline hs-CRP (mg/dL) & $0.6 \pm 0.9$ \\
\hline \multicolumn{2}{|l|}{ Anthropometrics } \\
\hline Height (cm) & $160.6 \pm 7.9$ \\
\hline Body weight (kg) & $60.3 \pm 9.7$ \\
\hline $\operatorname{BMI}\left(\mathrm{kg} / \mathrm{m}^{2}\right)$ & $23.3 \pm 3.0$ \\
\hline Body fat (\%) & $29.9 \pm 9.2$ \\
\hline \multicolumn{2}{|l|}{ Dietary intake } \\
\hline Energy (kcal/day) & $1554.5 \pm 494.5$ \\
\hline Protein (g/day) & $61.6 \pm 23.9$ \\
\hline Total fat (g/day) & $61.9 \pm 27.9$ \\
\hline Meat and beans (serving/day) & $5.1 \pm 3.0$ \\
\hline Vegetables (serving/day) & $1.9 \pm 1.5$ \\
\hline Fruits (serving/day) & $0.9 \pm 1.0$ \\
\hline Oil (serving/day) & $3.7 \pm 3.0$ \\
\hline Folate ( $\mu \mathrm{g} /$ day $)$ & $435.8 \pm 204.0$ \\
\hline Vitamin $B_{2}$ (mg/day) & $1.5 \pm 0.8$ \\
\hline Vitamin $B_{6}(\mathrm{mg} /$ day $)$ & $2.1 \pm 1.1$ \\
\hline Vitamin $B_{12}(\mu \mathrm{g} /$ day $)$ & $4.7 \pm 3.1$ \\
\hline \multicolumn{2}{|l|}{ Supplement } \\
\hline Folic acid, n (\%) & $108(78.3 \%)$ \\
\hline B-complex, n (\%) & $96(69.6 \%)$ \\
\hline \multicolumn{2}{|l|}{ Muscle mass } \\
\hline SMM (kg) & $22.6 \pm 4.5$ \\
\hline $\operatorname{SMM}_{\mathrm{Ht} 2}\left(\mathrm{~kg} / \mathrm{m}^{2}\right)$ & $8.7 \pm 1.2$ \\
\hline $\mathrm{SMM}_{\mathrm{Wt}}(\%)$ & $37.7 \pm 5.6$ \\
\hline \multicolumn{2}{|l|}{ Muscle function ${ }^{a}$} \\
\hline Hand grip strength (kg) & $21.1 \pm 7.4$ \\
\hline Arm lean mass (kg) & $1.7 \pm 0.5$ \\
\hline Muscle quality & $10.0 \pm 3.0$ \\
\hline
\end{tabular}

TABLE 1: Continued.

\begin{tabular}{lc}
\hline Variables & All $(\mathrm{n}=138)$ \\
\hline Others & \\
nPNA (g/kg) & $1.3 \pm 0.4$ \\
GNRI & $98.6 \pm 7.1$ \\
MET (kcal/day) & $753.8 \pm 311.7$ \\
\hline
\end{tabular}

Values are expressed as mean \pm standard deviation or number of patients (percentage).

${ }^{\mathrm{a}}$ Sample of 87 patients with the data of muscle function was analyzed. SD, standard deviation; CVD, cardiovascular disease; FPG, fasting plasma glucose; HOMA-IR, homoeostasis model assessment-estimated insulin resistance; TSH, thyroid-stimulating hormone; hs-CRP, high sensitivity Creactive protein; $\mathrm{BMI}$, body mass index; SMM, skeletal muscle mass; $\mathrm{Ht}$, height; Wt, weight; nPNA, normalized protein nitrogen appearance; GNRI, geriatric nutritional risk index; MET, metabolic equivalent.

years and above. Besides, higher Hcy was marginally associated with muscle function decline in hyperhomocysteinemia hemodialysis patients aged 65 years and above. A previous longitudinal study has confirmed the association between higher homocysteine lower muscle strength in a general population aged 50 years or older [22]. Contributing factors such as age, low physical performance, and the presence of comorbidities were associated with muscle wasting [23].

The previous studies showed that elevated Hcy is associated with muscle function declined in the older adult $[15,16]$. In addition to multiple hypotheses hyperhomocysteinemia impaired muscle function concluded by Veeranki and Tyagi [14], Hcy thiolactone reacts with proteins by a mechanism involving homocysteinylation of protein lysine residues in human serum and leads to protein damage and atrophy of skeletal muscle [24], the phenomenon called protein homocysteinylation [24]. Protein homocysteinylation is the posttranslational acylation of free amino groups (e.g., protein-Nhomocysteinylation) or formation of a covalent-S-S-bond (protein-S-homocysteinylation) mediated by Hcy thiolactone [24], which potentially causes significant alterations in the protein function. A study reported that protein-Nhomocysteinylation and protein-S-homocysteinylation were significantly higher in hemodialysis patients, and the significant association between plasma Hcy and protein-Shomocysteinylation was also found [25]. This indicated that hyperhomocysteinemia may cause the toxicity via the oxidative damage to proteins [26] and may result in muscle weakness and atrophy [24].

In our study, Kt/V was significantly associated with the lower SMM in hemodialysis patients. The negative association between Kt/V and muscle mass was also demonstrated in 34 Japanese hemodialysis patients [27]. The result indicated that patients with lower muscle mass may require a higher dialysis clearance. Therefore, muscle mass should be addressed while evaluating the hemodialysis adequacy.

The present study showed that dialysis vintage, creatinine, and BMI were independently associated with muscle mass and/or muscle function. The predialysis serum creatinine level will be proportional to dietary protein intake and the SMM that lower muscle mass may indicate the lower creatinine excretion [28]. The previous study showed that 


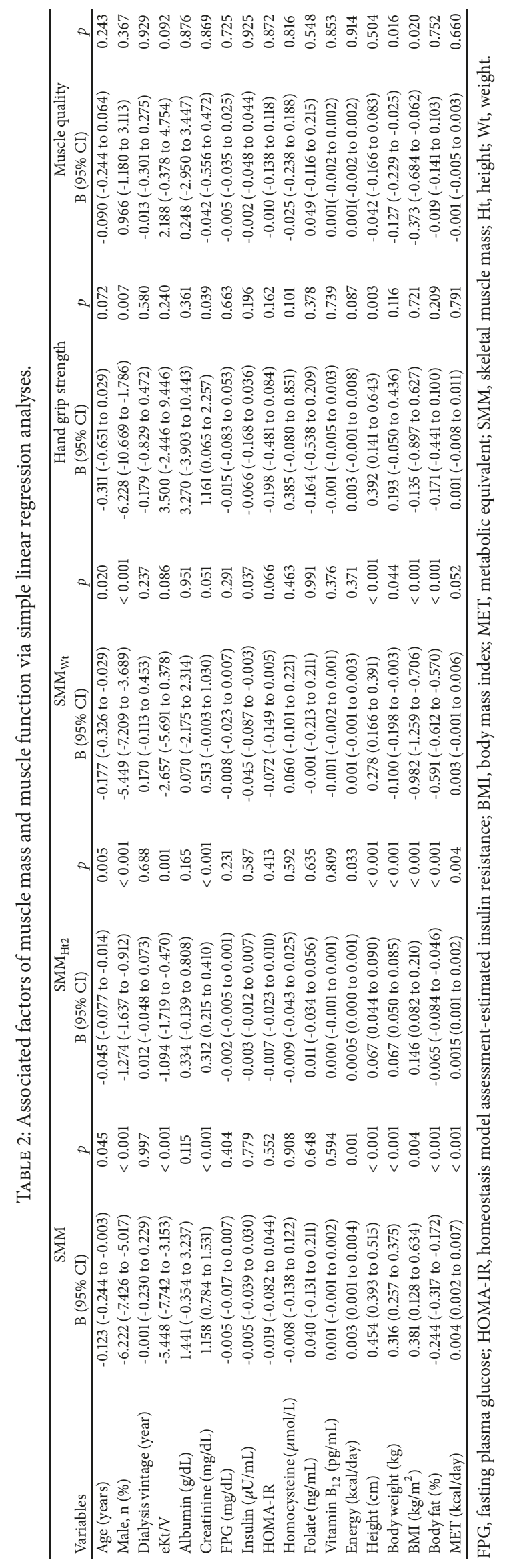


TABLE 3: Stepwise regression for determining muscle mass and muscle function in hemodialysis patients.

\begin{tabular}{|c|c|c|c|}
\hline Variables & $\mathrm{B}(95 \% \mathrm{CI})^{\mathrm{a}}$ & $p$ & $\overline{\text { adjusted } \mathrm{R}^{2}}$ \\
\hline SMM & & & 0.82 \\
\hline $\mathrm{Kt} / \mathrm{V}$ & $-1.13(-2.28$ to 0.03$)$ & 0.056 & \\
\hline Energy (kcal/day) & 0.0008 (0.0001 to 0.0015$)$ & 0.034 & \\
\hline $\operatorname{BMI}\left(\mathrm{kg} / \mathrm{m}^{2}\right)$ & $1.03(0.88$ to 1.19$)$ & $<0.001$ & \\
\hline Body fat (\%) & $-0.37(-0.42$ to -0.31$)$ & $<0.001$ & \\
\hline MET (kcal/day) & $0.0009(-0.0002$ to 0.0020$)$ & 0.102 & \\
\hline $\mathrm{SMM}_{\mathrm{Ht} 2}$ & & & 0.50 \\
\hline Homocysteine $(\mu \mathrm{mol} / \mathrm{L})$ & $-0.02(-0.05$ to 0.01$)$ & 0.060 & \\
\hline Creatinine (mg/dL) & $0.23(0.14$ to 0.31$)$ & $<0.001$ & \\
\hline Body fat (\%) & $-0.04(-0.06$ to -0.03$)$ & $<0.001$ & \\
\hline $\mathrm{SMM}_{\mathrm{Wt}}$ & & & 0.97 \\
\hline Homocysteine $(\mu \mathrm{mol} / \mathrm{L})$ & $-0.03(-0.06$ to -0.01$)$ & 0.020 & \\
\hline Creatinine $(\mathrm{mg} / \mathrm{dL})$ & $0.13(0.03$ to 0.22$)$ & 0.009 & \\
\hline Body fat (\%) & $-0.57(-0.59$ to -0.55$)$ & $<0.001$ & \\
\hline Hand grip strength & & & 0.35 \\
\hline Creatinine (mg/dL) & $0.93(0.18$ to 1.68$)$ & 0.015 & \\
\hline Energy (kcal/day) & $0.0023(-0.0004$ to 0.0050$)$ & 0.092 & \\
\hline Body fat (\%) & $-0.14(-0.31$ to 0.03$)$ & 0.101 & \\
\hline Muscle quality $^{2}$ & & & 0.18 \\
\hline Dialysis vintage (year) & $-0.15(-0.35$ to 0.05$)$ & 0.145 & \\
\hline $\mathrm{Kt} / \mathrm{V}$ & $1.91(-0.08$ to 3.91$)$ & 0.060 & \\
\hline Creatinine (mg/dL) & $0.45(0.06$ to 0.83$)$ & 0.023 & \\
\hline $\operatorname{BMI}\left(\mathrm{kg} / \mathrm{m}^{2}\right)$ & $-0.58(-0.88$ to -0.29$)$ & $<0.001$ & \\
\hline Body fat (\%) & $0.07(-0.04$ to 0.18$)$ & 0.190 & \\
\hline
\end{tabular}

${ }^{a}$ The significance levels of any potential factor for entry (SLE) and for stay (SLS) in the stepwise variable selection were set to 0.2. Age and gender were adjusted. Variables retained in the model are presented in the table.

BMI, body mass index; MET, metabolic equivalent; SMM, skeletal muscle mass; $\mathrm{Ht}$, height; $\mathrm{Wt}$, weight.

the hemodialysis vintage was negatively associated with the loss of SMM and muscle function. In addition, BMI was positively associated with SMM [27]. The possible reason for the negative correlation between BMI and MQ was supposed to be the calculation formula, BMI would be increase, and MQ would be decreased when skeletal muscle mass increases.

Our study shows that body fat was an independent determinant of muscle mass and muscle function among hemodialysis patients. A previous study was conducted using the computed tomography (CT) to take mid-thigh muscle cross-sectional area in a 36-year-old hemodialysis patient and an 80-year-old hemodialysis patient. Results showed that the fat accumulated in the striated muscle cells and intermuscular adipose tissue in older hemodialysis patients. Besides, the results also reveal that reduced muscle cross-sectional area, increased intermuscular adipose tissue, and/or intramuscular lipid were independently associated with aging and indices of maximal strength and physical performance [29].

Our study was with a number of strengths and limitations. The plasma homocysteine was analyzed by the international standard protocol in the hospital. We used the bioelectrical impedance analysis (BIA) as body composition measurement. The BIA is inexpensive, portable, and easyto-use as compared with others devices. The measurement conducted by BIA might be minor differences as compared with other methods such as the dual-energy X-ray absorptiometry (DEXA), CT, and magnetic resonance imaging
(MRI). However, the examination of total-body muscle mass using the BIA was highly correlated with estimation using the MRI in hemodialysis patients [30]. Finally, the basis of a cross-sectional design cannot provide the causal evidence between plasma Hcy and muscle mass. Therefore, the results must be interpreted with caution. Future cohort designs or control trials are suggested.

\section{Conclusion}

The current study revealed that hyperhomocysteinemia is high prevalence in hemodialysis patients. Elevated homocysteine level is associated with lower muscle mass in hemodialysis patients aged 65 years and above. Thus, the homocysteinelowering therapy might have a positive impact on muscle mass. The longitudinal studies are suggested to provide a deeper understanding of the association. Randomized control trials are needed for evaluating the effectiveness of therapy.

\section{Data Availability}

Data is available upon request to corresponding author of this article. 
TABLE 4: Stepwise regression analyses for determining muscle mass and muscle function in hemodialysis patients with hyperhomocysteinemia.

\begin{tabular}{|c|c|c|c|}
\hline Variables & $\mathrm{B}(95 \% \mathrm{CI})^{\mathrm{a}}$ & $p$ & adjusted $\mathrm{R}^{2}$ \\
\hline SMM & & & 0.84 \\
\hline Creatinine $(\mathrm{mg} / \mathrm{dL})$ & $0.23(0.01$ to 0.45$)$ & 0.042 & \\
\hline BMI $\left(\mathrm{kg} / \mathrm{m}^{2}\right)$ & $1.02(0.86$ to 1.18$)$ & $<0.001$ & \\
\hline Body fat (\%) & $-0.36(-0.41$ to -0.30$)$ & $<0.001$ & \\
\hline $\mathrm{SMM}_{\mathrm{Ht} 2}$ & & & 0.47 \\
\hline Creatinine $(\mathrm{mg} / \mathrm{dL})$ & $0.18(0.07$ to 0.29$)$ & 0.002 & \\
\hline Body fat (\%) & $-0.04(-0.06$ to -0.02$)$ & $<0.001$ & \\
\hline MET (kcal/day) & $0.0006(-0.0001$ to 0.0012$)$ & 0.084 & \\
\hline $\mathrm{SMM}_{\mathrm{Wt}}$ & & & 0.99 \\
\hline Homocysteine $(\mu \mathrm{mol} / \mathrm{L})$ & $-0.03(-0.05$ to -0.01$)$ & 0.027 & \\
\hline Creatinine $(\mathrm{mg} / \mathrm{dL})$ & $0.17(0.09$ to 0.25$)$ & $<0.001$ & \\
\hline Body fat (\%) & $-0.55(-0.56$ to -0.53$)$ & $<0.001$ & \\
\hline Hand grip strength & & & 0.35 \\
\hline Homocysteine $(\mu \mathrm{mol} / \mathrm{L})$ & $-0.41(-0.83$ to 0.01$)$ & 0.058 & \\
\hline Dialysis vintage (year) & $-0.40(-0.93$ to 0.14$)$ & 0.143 & \\
\hline $\mathrm{eKt} / \mathrm{V}$ & $6.65(1.39$ to 11.91$)$ & 0.014 & \\
\hline Creatinine $(\mathrm{mg} / \mathrm{dL})$ & 1.34 (0.37 to 2.32$)$ & 0.008 & \\
\hline Energy (kcal/day) & $0.0042(0.0008$ to 0.0075$)$ & 0.015 & \\
\hline Body fat (\%) & $-0.19(-0.40$ to 0.02$)$ & 0.079 & \\
\hline Muscle quality ${ }^{2}$ & & & 0.19 \\
\hline Homocysteine $(\mu \mathrm{mol} / \mathrm{L})$ & $-0.18(-0.37$ to 0.02$)$ & 0.071 & \\
\hline Dialysis vintage (year) & $-0.22(-0.46$ to 0.02$)$ & 0.068 & \\
\hline $\mathrm{eKt} / \mathrm{V}$ & $3.14(0.76$ to 5.53$)$ & 0.011 & \\
\hline Creatinine $(\mathrm{mg} / \mathrm{dL})$ & $0.54(0.08$ to 1.01$)$ & 0.023 & \\
\hline Energy (kcal/day) & $0.0015(0.00005$ to 0.0031$)$ & 0.045 & \\
\hline BMI $\left(\mathrm{kg} / \mathrm{m}^{2}\right)$ & $-0.47(-0.73$ to -0.21$)$ & $<0.001$ & \\
\hline
\end{tabular}

${ }^{a}$ The significance levels of any potential factor for entry (SLE) and for stay (SLS) in the stepwise variable selection were set to 0.2. Age and gender were adjusted. Variables retained in the model are presented in the table.

BMI, body mass index; MET, metabolic equivalent; SMM, skeletal muscle mass; $\mathrm{Ht}$, height; $\mathrm{Wt}$, weight.

\section{Disclosure}

The funder had no role in the decision to collect data, data analysis, or reporting of the results.

\section{Conflicts of Interest}

The authors had no conflicts of interest relevant to this article to be disclosed.

\section{Authors' Contributions}

Chi-Sin Wang and Tuyen Van Duong contributed equally to this paper.

\section{Acknowledgments}

The authors express the appreciation to medical staff and patients from Taipei Medical University Hospital, Wan-Fang Hospital, Shuang Ho Hospital, Cathay General Hospital, and Taipei Tzu-Chi Hospital, Wei-Gong Memorial Hospital, and Lutong Poh-Ai Hospital. The research was funded by
Ministry of Science and Technology in Taiwan (NSC-1022320-B-038-026; MOST 105-2320-B-038-033-MY3).

\section{References}

[1] United States Renal Data System, "2014 USRDS annual data report: Epidemiology of kidney disease in the United States," National Institutes of Health, National Institute of Diabetes and Digestive and Kidney Diseases, Bethesda, MD, 2014.

[2] A. J. Cruz-Jentoft, J. P. Baeyens, J. M. Bauer et al., "Sarcopenia: European consensus on definition and diagnosis," Age and Ageing, vol. 39, no. 4, Article ID afq034, pp. 412-423, 2010.

[3] F. Lamarca, J. J. Carrero, J. C. D. Rodrigues, F. G. Bigogno, R. L. Fetter, and C. M. Avesani, "Prevalence of sarcopenia in elderly maintenance hemodialysis patients: The impact of different diagnostic criteria," The Journal of Nutrition, Health \& Aging, vol. 18, no. 7, pp. 710-717, 2014.

[4] M.-T. Liao, C.-C. Sung, K.-C. Hung, C.-C. Wu, L. Lo, and K.-C. $\mathrm{Lu}$, "Insulin resistance in patients with chronic kidney disease," Journal of Biomedicine \& Biotechnology, 2012.

[5] T. A. Ikizler, N. J. Cano, H. Franch et al., "Prevention and treatment of protein energy wasting in chronic kidney disease patients: A consensus statement by the International Society of 
Renal Nutrition and Metabolism," Kidney International, vol. 84, no. 6, pp. 1096-1107, 2013.

[6] K. M. Majchrzak, L. B. Pupim, K. Chen et al., "Physical activity patterns in chronic hemodialysis patients: comparison of dialysis and nondialysis days," Journal of Renal Nutrition, vol. 15 , no. 2, pp. 217-224, 2005.

[7] H. Nishiwaki, T. Hasegawa, M. Shinji et al., "Practice pattern of physician's directions of exercise restriction in patients with chronic kidney disease: results from the Chronic Kidney Disease Japan Cohort study," Clinical and Experimental Nephrology, vol. 22, no. 5, pp. 1108-1115, 2018.

[8] K. Hiraki, T. Yasuda, C. Hotta et al., "Decreased physical function in pre-dialysis patients with chronic kidney disease," Clinical and Experimental Nephrology, vol. 17, no. 2, pp. 225-231, 2013.

[9] E. Sterky and B. G. Stegmayr, "Elderly patients on haemodialysis have 50\% less functional capacity than gender- and agematched healthy subjects," Scandinavian Journal of Urology, vol. 39, no. 5, pp. 423-430, 2005.

[10] U. V. Kharlamova and O. E. Ilyicheva, "Effect of homocysteine on left ventricular structural and functional parameters in patients on programmed hemodialysis," Terapevticheskii Arkhiv, vol. 85, no. 3, pp. 90-93, 2013.

[11] M. Sagheb, M. Ostovan, Z. Sohrabi, E. Atabati, G. Raisjalai, and J. Roozbeh, "Hyperhemocysteinemia and cardiovascular risks in hemodialysis patients," Saudi Journal of Kidney Diseases and Transplantation, vol. 21, no. 5, pp. 863-866, 2010.

[12] K. M. A. Swart, N. M. Van Schoor, M. W. Heymans, L. A. Schaap, M. Den Heijer, and P. Lips, "Elevated homocysteine levels are associated with low muscle strength and functional limitations in older persons," The Journal of Nutrition, Health \& Aging, vol. 17, no. 6, pp. 578-584, 2013.

[13] K. M. A. Swart, A. W. Enneman, J. P. van Wijngaarden et al., "Homocysteine and the methylenetetrahydrofolate reductase $677 \mathrm{C}->\mathrm{T}$ polymorphism in relation to muscle mass and strength, physical performance and postural sway," European Journal of Clinical Nutrition, vol. 67, no. 7, pp. 743-748, 2013.

[14] S. Veeranki and S. C. Tyagi, "Defective homocysteine metabolism: Potential implications for skeletal muscle malfunction," International Journal of Molecular Sciences, vol. 14, no. 7, pp. 15074-15091, 2013.

[15] H.-K. Kuo, K.-C. Liao, S. G. Leveille et al., "Relationship of homocysteine levels to quadriceps strength, gait speed, and latelife disability in older adults," The Journals of Gerontology. Series A, Biological Sciences and Medical Sciences, vol. 62, no. 4, pp. 434-439, 2007.

[16] T.-P. Ng, K. C. Y. Aung, L. Feng, S. C. Scherer, and K. B. Yap, "Homocysteine, folate, vitamin B-12, and physical function in older adults: Cross-sectional findings from the Singapore longitudinal ageing study," American Journal of Clinical Nutrition, vol. 96, no. 6, pp. 1362-1368, 2012.

[17] Y. M. Liou, C. J. C. Jwo, K. G. Yao, L.-C. Chiang, and L.-H. Huang, "Selection of appropriate chinese terms to represent intensity and types of physical activity terms for use in the Taiwan version of IPAQ," Journal of Nursing Research, vol. 16, no. 4, pp. 252-263, 2008.

[18] T. Wong, Y. Chen, P. Wu et al., "Ratio of dietary n-6/n-3 polyunsaturated fatty acids independently related to muscle mass decline in hemodialysis patients," PLoS ONE, vol. 10, no. 10, p. e0140402, 2015.
[19] M. F. Crutchlow, B. Robinson, B. Pappachen et al., "Validation of steady-state insulin sensitivity indices in chronic kidney disease," Diabetes Care, vol. 30, no. 7, pp. 1813-1818, 2007.

[20] K/DOQI Workgroup, "K/DOQI Clinical Practice Guidelines for Nutrition in Chronic Renal Failure," American Journal of Kidney Diseases, vol. 35, 2, no. 6, pp. S1-S140, 2000.

[21] S. Barbat-Artigas, Y. Rolland, M. Zamboni, and M. AubertinLeheudre, "How to assess functional status: A new muscle quality index," The Journal of Nutrition, Health \& Aging, vol. 16, no. 1, pp. 67-77, 2012.

[22] M. L. Vidoni, K. Pettee Gabriel, S. T. Luo, E. M. Simonsick, and R. S. Day, "Relationship between homocysteine and muscle strength decline: the baltimore longitudinal study of aging," The Journals of Gerontology. Series A, Biological Sciences and Medical Sciences, vol. 73, no. 4, pp. 546-551, 2018.

[23] S. Ohkawa, M. Odamaki, N. Ikegaya, I. Hibi, K. Miyaji, and H. Kumagai, "Association of age with muscle mass, fat mass and fat distribution in non-diabetic haemodialysis patients," Nephrology Dialysis Transplantation, vol. 20, no. 5, pp. 945-951, 2005.

[24] H. Jakubowski, "Homocysteine thiolactone: Metabolic origin and protein homocysteinylation in humans," Journal of Nutrition, vol. 130, no. 2S, pp. 377S-381S, 2000.

[25] A. F. Perna, E. Satta, F. Acanfora, C. Lombardi, D. Ingrosso, and N. G. De Santo, "Increased plasma protein homocysteinylation in hemodialysis patients," Kidney International, vol. 69, no. 5, pp. 869-876, 2006.

[26] D. Xu, R. Neville, and T. Finkel, "Homocysteine accelerates endothelial cell senescence," FEBS Letters, vol. 470, no. 1, pp. 20 24, 2000.

[27] Y. Morishita, K. Kubo, Y. Haga et al., "Skeletal Muscle Loss Is Negatively Associated With Single-Pool Kt/V and Dialysis Duration in Hemodialysis Patients," Therapeutic Apheresis and Dialysis, vol. 18, no. 6, pp. 612-617, 2014.

[28] L. H. Oterdoom, R. T. Gansevoort, J. P. Schouten, P. E. de Jong, R. O. B. Gans, and S. J. L. Bakker, "Urinary creatinine excretion, an indirect measure of muscle mass, is an independent predictor of cardiovascular disease and mortality in the general population," Atherosclerosis, vol. 207, no. 2, pp. 534-540, 2009.

[29] B. Cheema, H. Abas, B. Smith et al., "Investigation of skeletal muscle quantity and quality in end-stage renal disease," Nephrology, vol. 15, no. 4, pp. 454-463, 2010.

[30] G. A. Kaysen, F. Zhu, S. Sarkar et al., "Estimation of totalbody and limb muscle mass in hemodialysis patients by using multifrequency bioimpedance spectroscopy," American Journal of Clinical Nutrition, vol. 82, no. 5, pp. 988-995, 2005. 


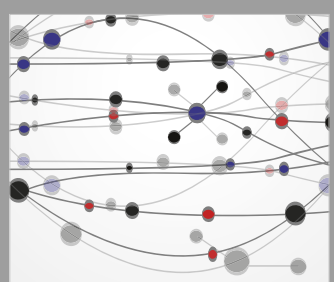

The Scientific World Journal
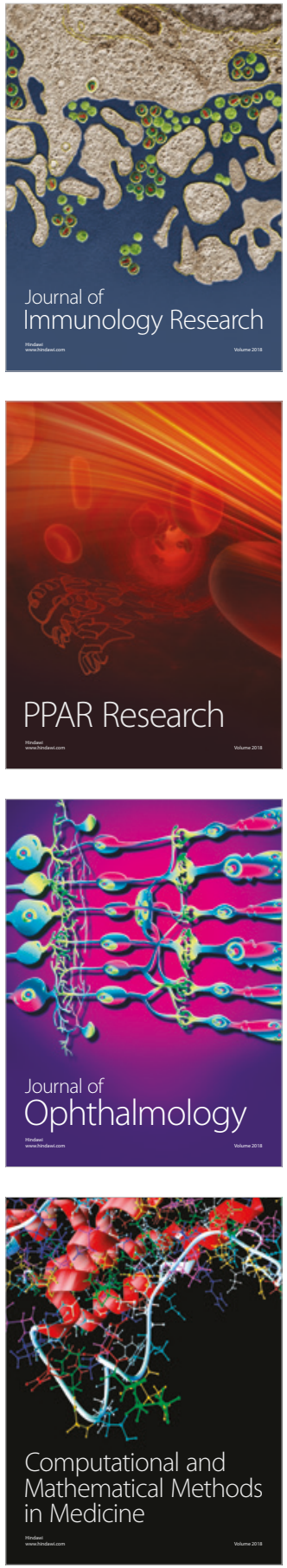

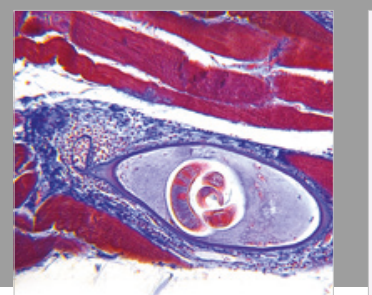

Gastroenterology Research and Practice

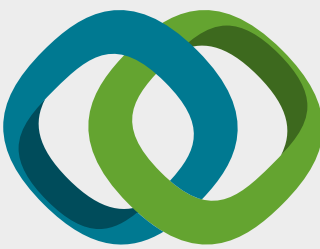

\section{Hindawi}

Submit your manuscripts at

www.hindawi.com
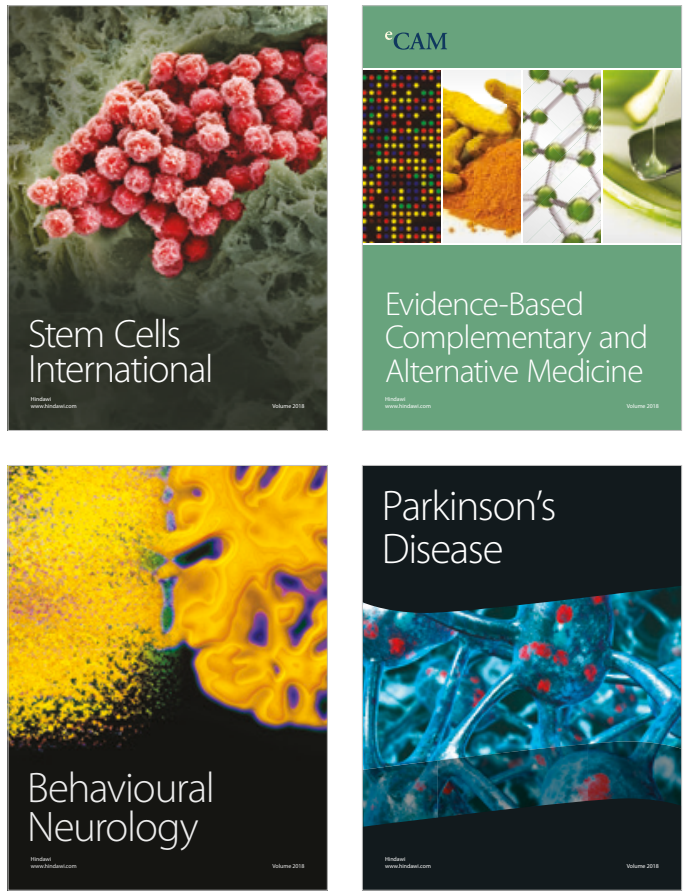

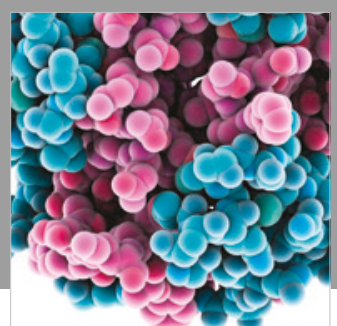

ournal of

Diabetes Research

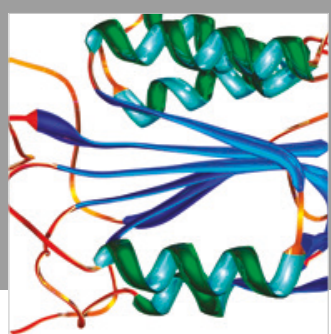

Disease Markers
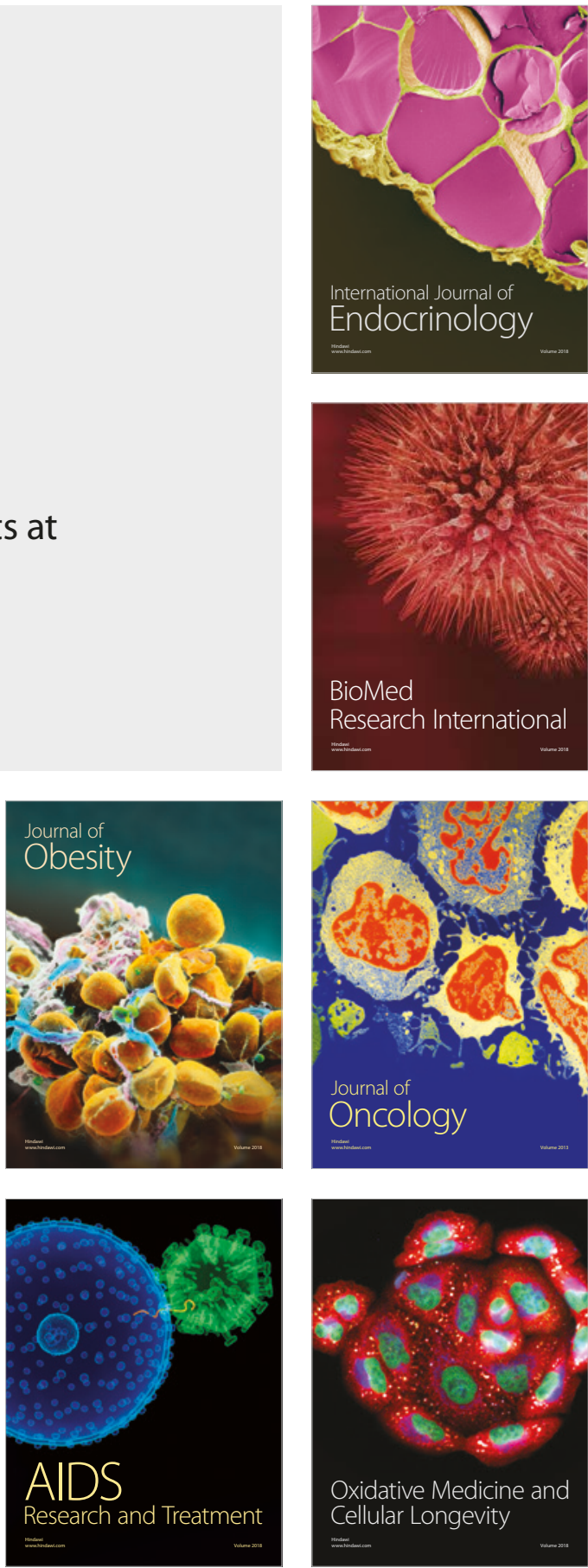